\title{
Tree-like tableaux
}

\section{Jean-Christophe Aval ${ }^{1}$, Adrien Boussicault ${ }^{1}$ and Philippe Nadeau ${ }^{2}$}

${ }^{1}$ LaBRI, Université Bordeaux 1, Talence, France

${ }^{2}$ Fakultät für Mathematik, Universität Wien, Vienna, Austria

\begin{abstract}
In this work we introduce and study tree-like tableaux, which are certain fillings of Ferrers diagrams in simple bijection with permutation tableaux and alternative tableaux. We exhibit an elementary insertion procedure on our tableaux which gives a clear proof that tableaux of size $n$ are counted by $n$ !, and which moreover respects most of the well-known statistics studied originally on alternative and permutation tableaux. Our insertion procedure allows to define in particular two simple new bijections between tree-like tableaux and permutations: the first one is conceived specifically to respect the generalized pattern 2-31, while the second one respects the underlying tree of a tree-like tableau.

Résumé. Dans ce travail nous introduisons et étudions les tableaux boisés, qui sont certains remplissages de diagrammes de Ferrers en bijection simple avec les tableaux de permutation et les tableaux alternatifs. Nous décrivons une procédure d'insertion élémentaire sur nos tableaux qui donne une preuve limpide que les tableaux de taille $n$ sont comptés par $n$ !, et qui de plus respecte la plupart des statistiques standard sur les tableaux de permutation et tableaux alternatifs. Notre procédure d'insertion permet en particulier de définir deux nouvelles bijections simples entre tableaux et permutations: la première est conçue spécifiquement pour respecter le motif généralisé 2-31 sur les permutations, tandis que la deuxième respecte l'arbre binaire sous-jacent à un tableau boisé.
\end{abstract}

Keywords: tree-like tableaux, permutation tableaux, alternative tableaux, permutations, binary trees

\section{Introduction}

Permutation tableaux and alternative tableaux are equivalent combinatorial objects that have been the focus of intense research in recent years. Originally introduced by Postnikov [Pos06], they were soon studied by numerous combinatorialists [Bur07, CN09, SW07, Wil05, Nad09, Vie08]. They also popped up surprisingly in order to get a combinatorial understanding of the equilibrium state of the PASEP model from statistical mechanics: this is the seminal work of Corteel and Williams, see [CW07a, CW07b. CW10].

In this work we introduce and study tree-like tableaux (cf. Definition 1.1), which are objects in simple bijection with alternative tableaux. Indeed, our results have immediate reformulations in terms of alternative/permutation tableaux (see Proposition 1.2). We chose to focus on these new tableaux for one main reason: they exhibit a natural tree structure (giving them their name: cf. Figure 2, right) more clearly than the alternative tableaux, and Section 4 stresses this particular view of considering the tableaux. As is mentioned in this last section, the present work originated in fact in the study of trees.

The main result of this work is Theorem 2.2. There is a simple bijective correspondence insertpoint between (1) tree-like tableaux of size $n$ together with an integer $i \in\{1, \ldots, n+1\}$ and (2) tree-like 
tableaux of size size $n+1$. A variation for symmetric tableaux insertpoint ${ }^{*}$ is also defined and shares similar properties, see Theorem 2.3 . We prove that insertpoint and insertpoint ${ }^{*}$ carry various statistics of tableaux in a straightforward manner: we obtain thus new easy proofs of formulas enumerating tableaux and symmetric tableaux, as well as information on the number of crossings (cf. Section 1.2).

An immediate corollary of Theorem 2.2 is that tree-like tableaux of size $n$ are enumerated by $n$ ! (and Theorem 2.2 implies that symmetric tableaux of size $2 n+1$ are enumerated by $2^{n} n$ !). Several bijections between tableaux and permutations appeared already in the literature; the ones that seem essentially distinct are [SW07] and the two bijections from [CN09]. All of them give automatically a correspondence as in Theorem 2.2, but none of them is as elementary as insertpoint. Conversely, it is clear that insertpoint allows to define various bijections between permutations and tableaux. We will focus on two of them here: the first one sends crossings to occurrences of the generalized pattern 2-31, while the second one preserves the binary trees naturally attached to permutations and tree-like tableaux.

Let us give a brief outline of this abstract. Section 1 introduces numerous definitions and notations, and most notably the tree-like tableaux which are the central focus of this work. Section 2 is the core section of this article: we introduce our main tool, the insertion insertpoint, and prove that it gives a 1-to- $(n+1)$ correspondence between tableaux of size $n$ and $n+1$. We use it to give elementary proofs of refined enumeration formulas for tableaux as well as symmetric tableaux, for which we use a modified insertion insertpoint ${ }^{*}$. Section 3 is centered around the enumeration of crossings; in particular, we use insertpoint to define a bijection between tableaux and permutations which sends crossings to occurrences of the pattern 2-31. In Section 4 we focus on the tree structure associated to a tree-like tableau, and give in particular another bijection between tableaux and permutations. We conclude in Section 5 by listing some possible extensions of the work presented here.

\section{Definitions and Notation}

\subsection{Ferrers diagrams, permutation and trees}

Ferrers diagrams: A Ferrers diagram $F$ is a left aligned finite set of unit cells in $\mathbb{Z}^{2}$, in decreasing number from top to bottom, considered up to translation: see Figure 1. left. The half-perimeter of $F$ is the sum of its number of rows plus its number of columns; it is also equal to the number of boundary edges, which are the edges found on the Southeast border of the diagram. We will also consider boundary cells, which are the cells of $F$ with no other cells to their Southeast.

There is a natural Southwest to Northeast order on boundary edges, as well as on boundary cells. Moreover, by considering the Southeast corner of boundary cells, these corners are naturally intertwined with boundary edges: we will thus speak of a boundary cell being Southwest or Northeast of a boundary edge. Two cells are adjacent if they share an edge.

Given two Ferrers diagrams $F_{1} \subseteq F_{2}$, we say that the set of cells $S=F_{2}-F_{1}$ (set-theoretic difference) is a ribbon if it is connected (with respect to adjacency) and contains no $2 \times 2$ square. In this case we say that $S$ can be added to $F_{1}$, or that it can be removed from $F_{2}$. Note that a removable ribbon from $F$ is equivalently a connected set $S$ of boundary cells of $F$, such that the Southwest-most cell of $S$ has no cell of $F$ below it, and the Northeast-most cell of $S$ has no cell of $F$ to its right.

Row/Column insertion: Let $F$ be a Ferrers diagram and $e$ one of its boundary edges. If $e$ is at the end of a row $r$, we define the insertion of a column at $e$ to be the addition of a cell to $r$ and all rows above it; symmetrically, if $e$ is at the end of a column denoted by $c$, we can insert a row at $e$ by adding a cell to 


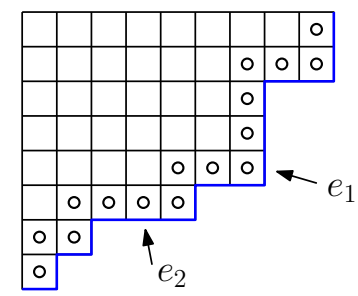

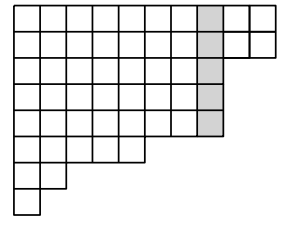

Insertion at $e_{1}$

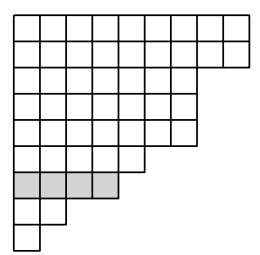

Insertion at $e_{2}$

Fig. 1: A Ferrers diagram of half perimeter 17 with its highlighted boundary cells and edges (left), and examples of insertions at the boundary edges $e_{1}$ and $e_{2}$.

$c$ and all columns to its left; see Figure 1, where the shaded cells of the figure are the added cells of the column or row.

Permutations and trees: We consider permutations $\sigma$ of $\{1, \ldots, n\}$, which are bijections from $\{1, \ldots, n\}$ to itself, and are counted by $n$ !. We will represent permutations as words $\sigma_{1} \ldots \sigma_{n}$ of length $n$ where $\sigma_{i}=\sigma(i)$. A descent is an index $i<n$ such that $\sigma_{i}>\sigma_{i+1}$. An occurrence of the pattern 2-31 in $\sigma$ is a pair $(i, j)$ of two indices such that $1 \leq i<j<n$ and $\sigma_{j+1}<\sigma_{i}<\sigma_{j}$.

A planar binary tree is a rooted tree such that each vertex has either two ordered children or no child; vertices with no child are called leaves, those of degree 2 are called nodes. The size of a tree is its number of nodes; see Figure 2 (right) for an example of tree of size 8.

\subsection{Tree-like tableaux}

We can now define the main object of this work:

Definition 1.1 (Tree-like tableau) $A$ tree-like tableau is a Ferrers diagram where each cell contains either 0 or 1 points (called respectively empty cell or pointed cell), with the following constraints:

(1) the top left cell of the diagram possesses a point, called the root point;

(2) for every non-root pointed cell c, there exists either a pointed cell above $c$ in the same column, or a pointed cell to its left in the same row, but not both;

(3) every column and every row possesses at least one pointed cell.

An example is shown on the left of Figure 2 Note that Condition (2) associates to each non-root point a unique other point; if we draw an edge between these two points, as well as an edge from every boundary edge to the closest point in its row or column, we create a binary tree, with nodes and leaves corresponding respectively to pointed cells and boundary edges. This is pictured on Figure 2, and explains the name given to our tableaux; we will come back to this tree structure with more detail in Section 4.

Let $T$ be a tree-like tableau. If the diagram of $T$ has half-perimeter $n+1$, then $T$ is easily seen to have exactly $n$ points: we let $n$ be the size of $T$, and we denote by $\mathcal{T}_{n}$ the set of tree-like tableaux of size $n$. A crossing of $T$ is an empty cell of $T$ with a point both above it and to its left; we let $\operatorname{cr}(T)$ be the number of crossings of $T$. The top points (respectively left points) of $T$ are the non-root points appearing in the first row (resp. the first column) of its diagram.

Alternative tableaux and permutation tableaux: Tree-like tableaux are closely related to alternative tableaux [Nad09, Vie08] as follows: given a tree-like tableau, change every non-root point $p$ to an arrow 

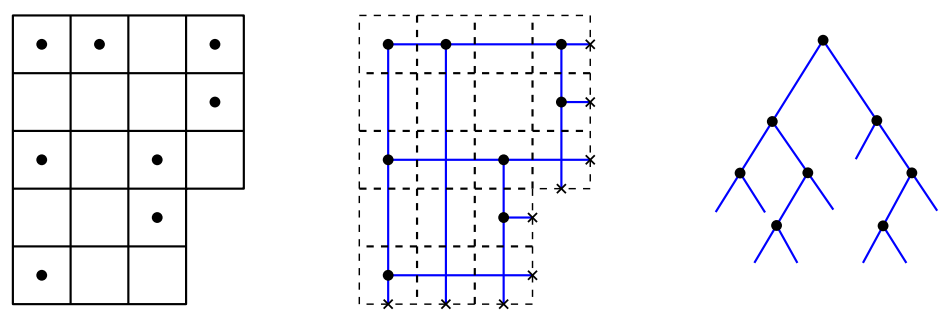

Fig. 2: A tree-like tableau (left) and the associated tree (right).

which is oriented left (respectively up) if there is no point left of $p$ (resp. above $p$ ). This transforms the tableau into a packed alternative tableau [Nad09, Section 2.1.2]. To obtain an alternative tableau, one simply deletes the first row and first column (empty rows and columns may then occur). We have more precisely the following correspondences, where we refer to [Nad09] for definitions on alternative and permutation tableaux:

Proposition 1.2 Let $n, i, j, k, \ell$ be nonnegative integers. There exist bijections between:

(1) Tree-like tableaux of half-perimeter $n+1$, with i left points, $j$ top points, $k$ rows, $\ell$ crossings.

(2) Permutation tableaux of half-perimeter $n$ with $i+1$ unrestricted rows, $j$ top ones, $k$ rows, $\ell$ superfluous ones.

(3) Alternative tableaux of half-perimeter $n-1$ with $i$ free rows, $j$ free columns, $k-1$ rows, $\ell$ free cells.

\section{The fundamental insertion}

This section is the core of this work. We describe a new way of inserting points in tree-like tableaux, shedding new light on numerous enumerative results on those tableaux.

\subsection{Main Result}

A key definition is the following one, which introduces a distinguished point in a tableau:

Definition 2.1 (Special point) Let $T$ be a tree-like tableau. The special point of $T$ is the Northeast-most point among those that occur at the bottom of a column.

This is well-defined since the bottom row of $T$ necessarily has a pointed cell (Definition 1.1. (3)), which is then at the bottom of a column.

Definition of insertpoint: Let $T$ be a tableau of size $n$ and $e$ be one of its boundary edges. We define first a tableau $T^{\prime}$ of size $n+1$ as follows: we insert a row or column at $e$, and mark with a point (the new point) the last cell of the row/column that was inserted. Let $T^{\prime}$ be the tableau obtained by this operation. Then we distinguish two cases:

(1) If $e$ is to the Northeast of the special point of $T$, then we simply define $i n \operatorname{sertpoint}(T, e):=T^{\prime}$;

(2) Otherwise, we add a ribbor ${ }^{(i)}$ of empty cells starting just to the right of the new point of $T^{\prime}$ and ending just below the special point of $T$. The result is tableau $T^{\prime \prime}$, and define $i n \operatorname{sertpoint}(T, e):=T^{\prime \prime}$.

(i) In the special case where $e$ is the lower edge of the special cell of $T$, define simply $T^{\prime \prime}=T^{\prime}$. 
The result is a tree-like tableau of size $n+1$, since all three conditions of Definition 1.1 are clearly satisfied. Examples of the two cases of insertpoint are given on Figure 3 Cells from the inserted rows/columns are shaded, while those from added ribbons are marked with a cross.

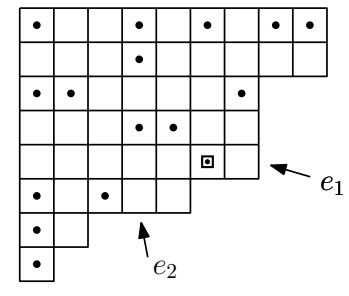

Tableau $T$

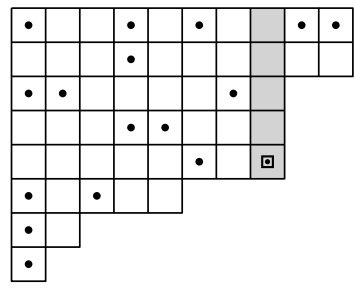

(1) $T_{1}=$ insertpoint $\left(T, e_{1}\right)$

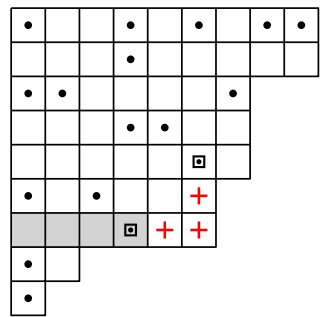

(2) $T_{2}=$ insertpoint $\left(T, e_{2}\right)$

Fig. 3: The two cases in the definition of insertpoint.

Theorem 2.2 For any $n \geq 1$ the insertion procedure insertpoint is a bijection between:

(A) The set of pairs $(T, e)$ where $T \in \mathcal{T}_{n}$ and $e$ is one of the $n+1$ boundary edges of $T$, and

(B) The set $\mathcal{T}_{n+1}$.

Proof: We first define a function removepoint; we will then prove that it is the desired inverse of insertpoint. Let $T$ be a tableau of size $n+1$ and consider the cell $c$ of its special point. In case there is a cell adjacent to the right of $c$ then follow the boundary cells to the Northeast of $c$ and let $c^{\prime}$ be the first cell encountered which has a point; we then remove the ribbon of empty cells comprised between $c$ and $c^{\prime}$. This leaves indeed a Ferrers diagram since $c^{\prime}$ is not the bottom cell of its column. Coming back to the general case, delete now the row or column which contains $c$ but no other points: let $T_{1}$ be the resulting tableau, and $e$ be the boundary edge of $T_{1}$ which is adjacent to $c$ in $T$. We define removepoint $(T):=\left(T_{1}, e\right) ; T_{1}$ has clearly size $n$ and $e$ is one of its boundary edges, and we claim removepoint is the desired inverse to the function insertpoint.

Let us now prove that insertpoint $\circ$ removepoint is the identity on $\mathcal{T}_{n+1}$. Let $T \in \mathcal{T}_{n+1}$, let $c$ be its special cell, and let $\left(T_{1}, e\right):=$ removepoint $(T)$.

- Suppose first that there is no cell in the diagram just to the right of $c$, that is, $c$ lies at the end of a row. In this case the special point of $T_{1}$ must be to the Southwest of $e$, therefore no ribbon will be added in insertpoint $\left(T_{1}, e\right)$ and this last tableau is thus clearly $T$.

- Now suppose there is a cell just to the right of $c$ : in this case the cell $c^{\prime}$ in the definition of removepoint contains the special point of $T_{1}$, since the removal of the ribbon will turn $c^{\prime}$ into a bottom cell of a column. Now $e$ will be to the left of $c^{\prime}$ in $T_{1}$, and so the application of insertpoint will add the removed ribbon: in this case also insertpoint $\left(T_{1}, e\right)=T$.

Conversely, let $T^{\prime}=\operatorname{insertpoint}(T, e)$ for $T \in \mathcal{T}_{n}$, and we wish to show that removepoint $\left(T^{\prime}\right)=$ $(T, e)$. The fundamental remark is that the new point $p$ added from $T$ to $T^{\prime}$ is the special point of $T^{\prime}$. The proof is as follows: if the insertion added no ribbon, then $p$ is inserted to the Northeast of the special point of $T$, and thus becomes the special point of $T^{\prime}$. If the insertion added a ribbon, then no column in $T^{\prime}$ between $p$ and the special point of $T$ has a bottom point because of this added ribbon, and thus once 
again $p$ is the special point of $T^{\prime}$. From this remark, it is immediate that removepoint $\left(T^{\prime}\right)=(T, e)$, and the proof is then complete.

Since $\left|\mathcal{T}_{1}\right|=1$ we have the immediate corollary: for $n \geq 1$ one has $\left|\mathcal{T}_{n}\right|=n$ !. We will give more precise enumerative results in Section 2.3

So we have an elementary proof that tableaux of size $n$ are equinumerous with permutations of length $n$. In fact, a multitude of bijections can be deduced from insertpoint; we will describe two such bijections in Sections 3.2 and 4.2

\subsection{Symmetric tableaux}

In this section we consider symmetric tableaux, i.e. tree-like tableaux which are invariant with respect to reflection through the main diagonal of their diagram. These are in bijection with symmetric alternative tableaux from [Nad09, Section 3.5], and "type B permutation tableaux" from [LW08]. The size of such a tableau is necessarily odd, and those of size $2 n+1$ (denoted by $\mathcal{T}_{2 n+1}^{\text {sym }}$ ) are counted by $2^{n} n$ ! (see [LW08, Nad09]); we will give a simple proof of this thanks to a modified insertion procedure.
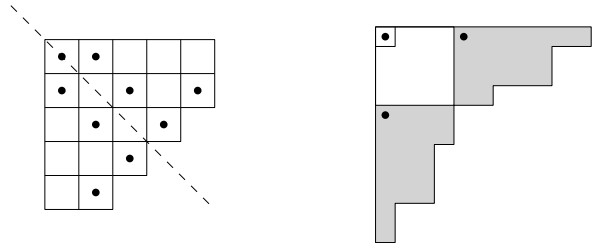

Fig. 4: A symmetric tableau, and the embedding of tree-like tableaux in symmetric tableaux.

Note that given a tree-like tableau $T$ of size $n$, one can associate to it a symmetric tableau as follows: if $T$ has $k$ columns, then add on top of it a $k \times k$ square of cells, where only the top left cell is pointed; then add to the right of the square the reflected tableau $T *$ : see Figure 4 right. In this way we embed naturally $\mathcal{T}_{n}$ in $\mathcal{T}_{2 n+1}^{\text {sym }}$.

We now define a modified point insertion insertpoint ${ }^{*}$ for symmetric tableaux. First let us call $*$ special point of a symmetric tableau the point at the bottom of a column which is Northeast-most among those that are Southwest of the diagonal.

Modified insertion: Let $T \in \mathcal{T}_{2 n+1}^{s y m}$ and $(e, \varepsilon)$ be a pair consisting of a boundary edge $e$ Southwest of the diagonal (there are $n+1$ such edges) and $\varepsilon \in\{+1,-1\}$. Insert first a row/column at $e$ with a point at the end, and insert also the symmetric column/row, to get a tableau $T^{\prime}$. There are then three cases:

(1) If $\varepsilon=+1$ and $e$ is Northeast of the $*$-special point, simply define insertpoint $^{*}(T, e,+1):=T^{\prime}$.

(2) If $\varepsilon=+1$ and $e$ is Southwest of the $*$-special point, add a ribbon to $T^{\prime}$ between the new point (Southwest of the diagonal) and the $*$-special point of $T$ below the diagonal; add also the symmetric ribbon. If $T^{\prime \prime}$ is the resulting tableau, then define insertpoint ${ }^{*}(T, e,+1):=T^{\prime \prime}$.

(3) If $\varepsilon=-1$, then add a ribbon in $T^{\prime}$ between the two new points, and the resulting tableau is by definition insertpoint $^{*}(T, e,-1)$.

Examples of all 3 cases are given on Figure 5 . 

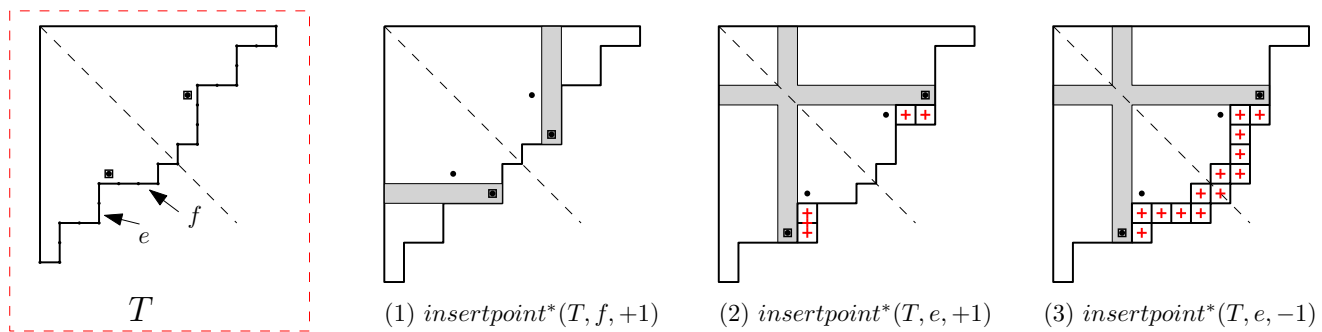

Fig. 5: The three cases in the definition of insertpoint*

Theorem 2.3 The modified point insertion insertpoint ${ }^{*}$ is a bijection between the set of triplets $(T, e, \varepsilon)$ as above and $\mathcal{T}_{2 n+3}^{\text {sym }}$.

Consequently, $\left|\mathcal{T}_{2 n+3}^{\text {sym }}\right|=2(n+1)\left|\mathcal{T}_{2 n+1}^{\text {sym }}\right|$ and thus $\left|\mathcal{T}_{2 n+1}^{\text {sym }}\right|=2^{n} n$ ! for $n \geq 0$.

Proof: The proof is similar to the one of Theorem 2.2 and is omitted. The main ingredient is that the new point added Southwest of the diagonal is the $*$-special point of the resulting tableau, which allows one to invert the insertion procedure.

It is easy to check that when only $\varepsilon=+1$ is chosen during insertions, then the symmetric tableaux obtained in this case are precisely those corresponding to the embedding of usual tree-like tableaux into symmetric ones defined above. Indeed insertpoint ${ }^{*}$ involves then only cases (1) and (2) which correspond to the usual insertion insertpoint.

\subsection{Enumerative consequences}

We now show how our insertion procedures give elementary proofs of some enumerative results on tableaux. Let $T_{n}(x, y)$ be the polynomial

$$
T_{n}(x, y)=\sum_{T \in \mathcal{T}_{n}} x^{\operatorname{left}(T)} y^{\operatorname{top}(T)},
$$

where left $(T)$ and top $(T)$ are respectively the number of left points and top points in $T$. When we insert a point in a tableau $T$ of size $n$, then we add a left (respectively right) point to it if the Southwest-most edge (resp. Northeast-most edges) is picked, while for other boundary edges the number of top and left points remains the same. This gives immediately the recurrence relation $T_{n+1}(x, y)=(x+y+n-1) T_{n}(x, y)$ which together with $T_{1}(x, y)=1$ gives:

$$
T_{n}(x, y)=(x+y)(x+y+1) \cdots(x+y+n-2) .
$$

This formula was proved in [CN09] and then bijectively in [Nad09]; the proof above is arguably the simplest one (and is bijective).

We can also give a generalization of Formula (1) to symmetric tableaux [LW08, CK10]. Following [CK10, Section 5] - reformulated in terms of tree-like tableaux - we let

$$
T_{2 n+1}^{\text {sym }}(x, y, z)=\sum_{T \in \mathcal{T}_{2 n+1}^{\text {sym }}} x^{\operatorname{left}(T)} y^{\operatorname{top}{ }^{*}(T)} z^{\operatorname{diag}(T)},
$$


where $\operatorname{diag}(T)$ is the number of crossings among the diagonal cells; for top* $(T)$, consider the northmost non-root point $p$ in the first column, then the number of points on the row of $p$ is by definition $\operatorname{top}^{*}(T)$. Let $T^{\prime}=$ insertpoint $^{*}(T, e, \varepsilon)$ be as in Theorem 2.3 One has $\operatorname{diag}\left(T^{\prime}\right)=\operatorname{diag}(T)+1$ when $\varepsilon=-1$, and $\operatorname{left}\left(T^{\prime}\right)=\operatorname{left}(T)+1$ when $e$ is the Southwest-most edge. Furthermore, if the row $r$ considered in the definition of top* $(T)$ has its boundary edge $e^{\prime}$ Southwest of the diagonal, then the insertion at $e=e^{\prime}$ increases $t o p^{*}(T)$ by one; otherwise, the column $c$ symmetric to $r$ ends below the diagonal at a boundary edge $e^{\prime \prime}$, and then the insertion at $e=e^{\prime \prime}$ increases $\operatorname{top}^{*}(T)$ by one. For all other choices of $e$ we have $\operatorname{top}^{*}\left(T^{\prime}\right)=\operatorname{top}(T)$. Therefore we obtain the recurrence formula $T_{2 n+3}^{s y m}(x, y, z)=(1+z)(x+y+n-$ 1) $T_{2 n+1}^{\text {sym }}(x, y, z)$, from which it follows:

$$
T_{2 n+1}^{\text {sym }}(x, y, z)=(1+z)^{n}(x+y)(x+y+1) \cdots(x+y+n-2) .
$$

Note that this proof is much simpler than any of the two proofs given in [CK10].

\section{Crossings and Permutations}

We study here the interplay between crossings in a tableau and the insertion insertpoint.

\subsection{Enumeration of crossings}

The starting point is the following:

Lemma 3.1 Let $T \in \mathcal{T}_{n}$. The crossings of $T$ are the cells of the ribbons added in its insertion history, i.e in the $n-1$ applications of insertpoint necessary to construct $T$ starting from the tableau of size 1.

Proof: In an application of insertpoint, the empty cells from a ribbon correspond clearly to crossings, while the empty cells from the row or column insertions are not crossings. Furthermore insertpoint does not modify crossings in the original tableau, which achieves the proof.

Let $T$ be a tableau of size $n$. From the Southwest to the Northeast, we label its boundary edges $e_{0}(T), \ldots, e_{n}(T)$ and its boundary cells $b_{0}(T), \ldots, b_{n-1}(T)$. We have the following proposition whose easy proof is omitted:

Proposition 3.2 Let $T \in \mathcal{T}_{n}, i \in\{0, \ldots, n\}$, and consider $T^{\prime}=\operatorname{insertpoint}\left(T, e_{i}\right)$. Then the special cell of $T^{\prime}$ is $b_{i}\left(T^{\prime}\right)$. Moreover, if $b_{k}(T)$ is the special cell of $T$ (where $0 \leq k \leq n-1$ ), then we have $\operatorname{cr}\left(T^{\prime}\right)=\operatorname{cr}(T)$ if $k \leq i$, while $\operatorname{cr}\left(T^{\prime}\right)=\operatorname{cr}(T)+(k-i)$ if $k>i$.
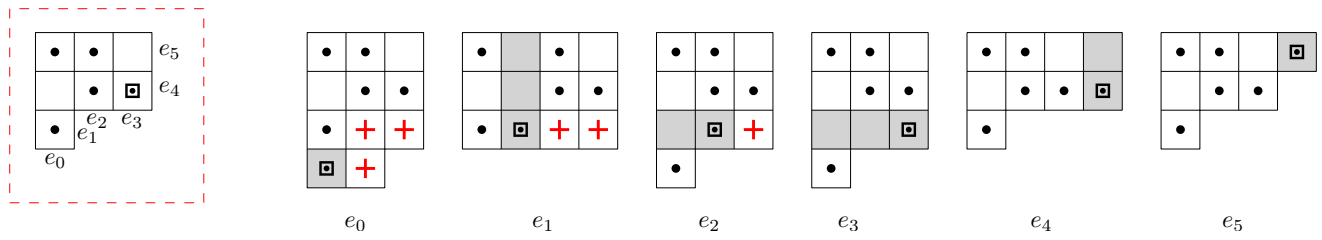

Fig. 6: A tableau of size 5 and all 6 possible point insertions. 
This is illustrated on Figure 6, for which $k=3$. A first consequence of the proposition is that given $k \in$ $\{0, \ldots, n-1\}$, there are $n ! / n=(n-1)$ ! tableaux $T$ in $\mathcal{T}_{n}$ where the special cell of $T$ is $b_{k}(T)$. A second consequence is that given such a tableau $T$, the total number of ribbon cells added when constructing all tableaux $T^{\prime}=\operatorname{insertpoint}\left(T, e_{i}\right)$ (for $i=0, \ldots, n$ ) is simply $1+2+\ldots+k=\left(\begin{array}{c}k+1 \\ 2\end{array}\right)$, and thus the total number of crossings in these $n+1$ tableaux is $(n+1) \operatorname{cr}(T)+\left(\begin{array}{c}k+1 \\ 2\end{array}\right)$. Let $C r_{n}=\sum_{T} c r(T)$ where $T$ runs through all tree-like tableaux of size $n$; the previous reasoning shows that for $n \geq 1$ :

$$
C r_{n+1}-(n+1) C r_{n}=(n-1) ! \times \sum_{k=0}^{n-1}\left(\begin{array}{c}
k+1 \\
2
\end{array}\right)=(n-1) !\left(\begin{array}{c}
n+1 \\
3
\end{array}\right)
$$

If we let $X_{n}=C r_{n} / n$ !, we obtain simply $X_{n+1}-X_{n}=(n-1) / 6$, from which we get:

Proposition 3.3 The total number of crossings in $\mathcal{T}_{n}$ is given by $C r_{n}=n ! \times(n-1)(n-2) / 12$.

This can also be stated as: given the uniform distribution on $\mathcal{T}_{n}$, the expectation of $\operatorname{cr}(T)$ is given by $(n-1)(n-2) / 12$. This was proved first in [CH07. Theorem 1] by a lengthy computation, which relied on the recursive construction of (permutation) tableaux obtained by adding the leftmost column.

\subsection{A first bijection with permutations}

As mentioned at the end of Section 2.1, it is immediate to construct bijections from $\mathcal{T}_{n}$ to permutations using insertpoint. We will here define one with the goal of sending crossings of tableaux to occurrences of the pattern 2-31 in a permutation.

First, a tableau $T$ of size $n$ is naturally encoded by a list of integers $a(T)=\left(a_{1}(T), \ldots, a_{n}(T)\right)$ satisfying $0 \leq a_{i}(T) \leq i-1$. This is done as follows: let $T_{1}, T_{2}, T_{3}, \ldots, T_{n}=T$ be the tableaux from the insertion history of $T$. For $i \in\{2, \ldots, n\}$, we define $a_{i}(T)$ as the index $j$ such that $i n s e r t p o i n t ~\left(T_{i-1}, e_{j}\right)=$ $T_{i}$, using the labeling of boundary edges defined before Proposition 3.2

This proposition tells us then that $c r(T)=\sum_{i=1}^{n-1} \max \left(a_{i}(T)-a_{i+1}(T), 0\right)$.

Now consider a word composed simply of $n$ letters $X$, which we will replace one by one by integers. For $i=n, n-1, \ldots, 1$ in succession, we do the following: among the $i$ remaining $X$ 's, pick the one which has $a_{i}(T)$ other $X$ 's to its left in the word, and replace it by the value $i$. For instance, if $a(T)=$ $(0,1,0,3,1)$, then the successive words are $X 5 X X X, X 5 X X 4,35 X X 4,35 X 24$, and finally 35124 . In general, if $\tau$ is the permutation obtained after this transformation, we define $\Phi_{1}(T):=\tau^{-1}$.

Theorem 3.4 $\Phi_{1}$ is a bijection from $\mathcal{T}_{n}$ to permutations of length $n$. If $\sigma=\Phi_{1}(T)$, then cr $(T)$ is equal to the number of occurrences of 2-31 in $\sigma$.

Proof: We will just give a sketch. First, it is clear that the construction is bijective. Now let $\Phi_{1}(T)=\tau^{-1}$ as above. Then note that in $\tau$, the value $i+1$ appears left of $i$ if and only if $a_{i}(T) \geq a_{i+1}(T)$, and that in this case there are $a_{i}(T)-a_{i+1}(T)$ values smaller than $i$ occurring between the positions occupied in $\tau$ by $i$ and $i+1$. This means that $a_{i}(T) \geq a_{i+1}(T)$ if and only if $i$ is a descent in $\Phi_{1}(T)\left(=\tau^{-1}\right)$, and in this case this descent stands for the ' 3 ' in $a_{i}(T)-a_{i+1}(T)$ occurrences of the pattern 2-31. The theorem follows then from the formula above expressing $\operatorname{cr}(T)$ in terms of $a(T)$. 
This bijection is much simpler than bijection II from [CN09], which was designed specifically to preserve the equivalent pattern 31-2.

\section{Trees and Tree-like tableaux}

\subsection{Trees from permutations and tableaux}

From permutations to binary trees: We define an increasing tree of size $n$ to be a binary tree of size $n$ where the $n$ nodes are labeled by all integers in $\{1, \ldots, n\}$ in such a way that the labels increase along the path from the root to any node. There is a well-known bijection with permutations: given an increasing tree $T$, traverse its vertices in inorder, which means recursively traverse the left subtree, then visit the root, then traverse the right subtree. By recording node labels in the order in which they are visited, one obtains the wanted permutation: see Figure 7(left). If $\sigma$ is a permutation with associated increasing tree inctree $(\sigma)$, then we define $\operatorname{tree}(\sigma)$ as the binary tree obtained by forgetting the labels in inctree $(T)$.

From tree-like tableaux to binary trees: We described this correspondence after Definition 1.1. It can also be obtained graphically by drawing two lines from every point of $T$, one down and one to the right, and stopping them at the boundary. We let $\operatorname{tree}(T)$ be the binary tree thus constructed, see Figure 7 (right). Note that there is a natural identification between boundary edges of $T$ and leaves of $\operatorname{tree}(T)$.
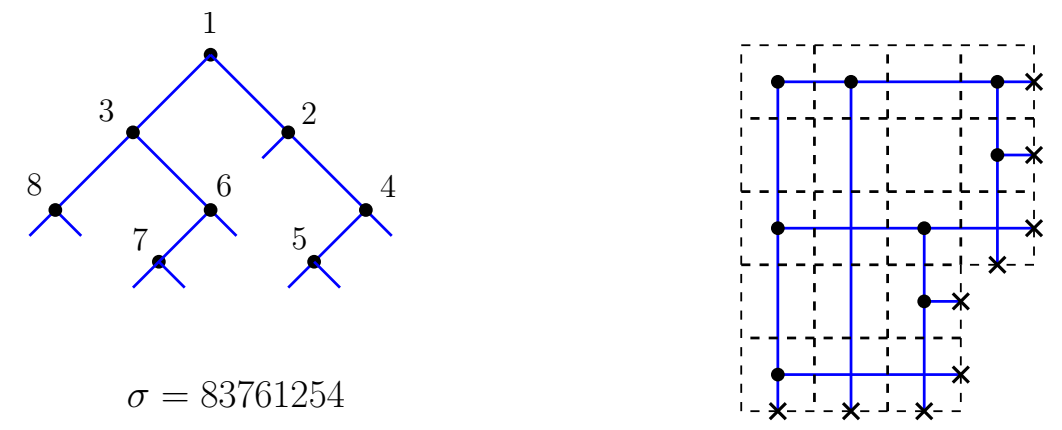

Fig. 7: The same binary tree arising from a permutation (left) and a tableau (right).

\subsection{The tree preserving bijection}

Using insertpoint, we now define a bijection $\Phi_{2}$ between permutations and tree-like tableaux which preserves the binary trees attached to the objects. For this we proceed by induction on $n$.

Let $\sigma$ be a permutation of size $n+1$, and $\tau$ be the permutation of size $n$ obtained by deleting $n+1$ in $\sigma$. By induction hypothesis, the tableau $T:=\Phi_{2}(\tau)$ is well defined and satisfies $\operatorname{tree}(T)=\operatorname{tree}(\tau)$. Define $L$ to be the leaf of inctree $(\tau)$ appearing in the inorder traversal at the position occupied by $n+1$ in $\sigma$ : then inctree $(\sigma)$ is obtained by replacing $L$ by a node labeled $n+1$ with two leaves. Now $L$ corresponds naturally to a boundary edge $e_{L}$ in $\Phi_{2}(\tau)$, and we define $\Phi_{2}(\sigma)=\operatorname{insertpoint}\left(\Phi_{2}(\tau), e_{L}\right)$.

Theorem 4.1 Given $n \geq 1$, the function $\Phi_{2}$ is a bijection between permutations of length $n$ and tree-like tableaux of size $n$, satisfying tree $(\sigma)=\operatorname{tree}\left(\Phi_{2}(\sigma)\right)$. 
This is a simple consequence of the properties of insertpoint. The permutation $\sigma$ and the tree-like tableau $T$ from Figure 7 satisfy $\Phi_{2}(\sigma)=T$.

Remark: The tree structure attached to tableaux is not new: first Burstein [Bur07] defined it on so-called bare tableaux, which are essentially our tree-like tableaux minus a column. Then this tree structure was also studied by the third author in some detail [Nad09, Section 4]. The main difference is that, although the (unlabeled) tree structure is essentially the same, the labeling is quite different: here we have a quite simple bijection with increasing trees, while the labelings from the two aforementioned references involve some complicated increasing/decreasing conditions. The root of such complication can be traced to the fact that the boundary edges in [Bur07, Nad09] were labeled independently of the structure of the tree, while here we use the tree to determine the labeling.

\subsection{Unambiguous trees}

As mentioned in the introduction, this work had its origin in problems about trees, and not tableaux; we will here briefly describe such a problem. Suppose we draw the nodes of a plane binary tree as points in the center of unit cells of $\mathbb{Z}^{2}$, where the children of a node are drawn below and to the right of this node (as in the trees tree $(T)$ attached to a tree-like tableau $T$ ); we allow edges to cross outside of nodes. Let us call the drawing unambiguous if, when one deletes the edges of the tree, it is then possible to reconstruct them uniquely: one sees that this comes down essentially to condition (2) in Definition 1.1 .

We are led to the following definition: an unambiguous tree is a tree-like tableau T such each corner of the diagram of $T$ contains a point (a corner is a cell with no cell adjacent below it or to its right). The connection is as follows: given an unambiguous drawing, consider the smallest Ferrers diagram that contains it: if this tableau contains no empty row or column (corresponding to a compact drawing), then we obtain exactly an unambiguous tree. We proved exact enumeration formulas for the number of unambiguous tree corresponding to a given binary tree, or the total number of unambiguous trees, which will appear in a future work.

\section{Further results and questions}

In this work we described a very simple insertion procedure insertpoint which can be seen as a 1-to$(n+1)$ correspondence between the sets $\mathcal{T}_{n}$ and $\mathcal{T}_{n+1}$. We proved that from this simple seed one could produce automatically most of the enumerative results known on tableaux, as well as design bijections to permutations with various properties. Other enumeration results can also be proved with the same techniques: enumeration of tableaux according to the number of rows (this gives Eulerian numbers [Wil05]), or the total number of cells. Another set of results which was not stated here for lack of space is the generalization of the constructions of Sections 3 and 4 to symmetric tableaux.

A further question would be to revisit the work of Corteel and Williams on the PASEP model from statistical mechanics (see [CW10, CW07b, CW07a]), which involves objects related to alternative tableaux. In particular, do their (weighted) staircase tableaux have recursive decompositions similar to those given here for tree-like tableaux ?

Finally, the insertion procedure makes it very easy to generate a tree-like tableau uniformly at random among those of size $n$. In fact (by using adapted structures for our objects), it is possible to generate such a tableau with space and memory in $O\left(n^{2}\right)$. A related point is the asymptotic behavior of tree-like tableaux. For instance, is there a "limit shape" to our objects, that is a certain curve to which the boundary 
of tableaux (rescaled by a factor $1 / n$ ) converge with high probability? A heuristic approach and early computer experiments tend to show that such a limit shape exists indeed, and is an arc of parabola; this is work in progress.

\section{Acknowledgements}

The authors thank Valentin Féray for insightful comments. The second author is grateful to Gérard $\mathrm{H}$. E. Duchamp and Christophe Tollu for useful discussions during the CIP seminar at Paris 13 university, discussions which were the starting point of this work.

\section{References}

[Bur07] Alexander Burstein. On some properties of permutation tableaux. Ann. Comb., 11(3-4):355368, 2007.

[CH07] Sylvie Corteel and Paweł Hitczenko. Expected values of statistics on permutation tableaux. In 2007 Conference on Analysis of Algorithms, AofA 07, Discrete Math. Theor. Comput. Sci. Proc., AH, pages 325-339. 2007.

[CK10] Sylvie Corteel and Jang Soo Kim. Combinatorics on permutation tableaux of type $A$ and type B. arXiv:1006.3812v1, 2010.

[CN09] Sylvie Corteel and Philippe Nadeau. Bijections for permutation tableaux. European J. Combin., 30(1):295-310, 2009.

[CW07a] Sylvie Corteel and Lauren K. Williams. A Markov chain on permutations which projects to the PASEP. Int. Math. Res. Not. IMRN, (17):Art. ID rnm055, 27, 2007.

[CW07b] Sylvie Corteel and Lauren K. Williams. Tableaux combinatorics for the asymmetric exclusion process. Adv. in Appl. Math., 39(3):293-310, 2007.

[CW10] Sylvie Corteel and Lauren K. Williams. Staircase tableaux, the asymmetric exclusion process, and Askey-Wilson polynomials. Proc. Natl. Acad. Sci. USA, 107(15):6726-6730, 2010.

[LW08] Thomas Lam and Lauren K. Williams. Total positivity for cominuscule Grassmannians. New York J. Math., 14:53-99, 2008.

[Nad09] Philippe Nadeau. The structure of alternative tableaux. arXiv:0908.4050v2, 2009.

[Pos06] Alexander Postnikov. Total positivity, Grassmannians, and networks. arXiv:math/0609764v1, 2006.

[SW07] Einar Steingrímsson and Lauren K. Williams. Permutation tableaux and permutation patterns. J. Combin. Theory Ser. A, 114(2):211-234, 2007.

[Vie08] Xavier Viennot. Alternative tableaux, permutations and partially asymmetric exclusion process. Slides of a talk at the Isaac Newton Institute in Cambridge, 2008.

[Wil05] Lauren K. Williams. Enumeration of totally positive Grassmann cells. Adv. Math., 190(2):319$342,2005$. 